\title{
High birefringence and low loss circular air-holes photonic crystal fiber using complex unit cells in cladding
}

\author{
Tzong-Jer Yang ${ }^{\mathrm{a}}$, Lin-Fang Shen ${ }^{\mathrm{b}}$, Yuan-Fong Chau ${ }^{\mathrm{c}, *}$, Ming-Je Sung ${ }^{\mathrm{d}}$, Daru Chen ${ }^{\mathrm{b}}$, Din Ping Tsai ${ }^{\mathrm{e}}$ \\ a Department of Electrical Engineering, Chung Hua University, Hsinchu, Taiwan, ROC \\ ${ }^{\mathrm{b}}$ Department of Information and Electronic Engineering, Zhejiang University, China \\ ${ }^{\mathrm{c}}$ Department of Electronic Engineering, Ching-Yun University, 229, Chien-Hsin Road, Jung-Li 320, Taiwan, ROC \\ ${ }^{\mathrm{d}}$ Graduate Institute of Photonics and Optoelectronics, National Taiwan University, Taiepi 106, Taiwan, ROC \\ e Department of Physics, National Taiwan University, Taiepi 106, Taiwan, ROC
}

\section{A R T I C L E I N F O}

\section{Article history:}

Received 21 February 2008

Received in revised form 7 May 2008

Accepted 7 May 2008

\section{Keywords:}

Birefringence

Finite-element method

Optical fiber

Photonic crystal fiber (PCF)

\begin{abstract}
A B S T R A C T
We propose a high birefringence and low loss index-guiding photonic crystal fiber (PCF) using the complex unit cells in cladding by the finite-element method. Results show that the birefringence and confinement loss in such PCF fiber is determined not only by the whole cladding asymmetry but also the shape of the PCF core. The maximal modal birefringence and lowest confinement loss of our proposed structures at the excitation wavelength of $\lambda=1550 \mathrm{~nm}$ can be achieved at $8.7 \times 10^{-3}$ and $5.27 \times 10^{-5} \mathrm{~dB} / \mathrm{km}$, respectively.
\end{abstract}

(c) 2008 Elsevier B.V. All rights reserved.

\section{Introduction}

In recent years, there has been a significant interest in the new technology of the photonic crystal fibers (PCFs) due to their wide range of unusual optical properties, such as endlessly single-mode operation, high birefringence, high nonlinearity and tailorable chromatic dispersion [1-11]. Among the features of PCFs, birefringence is one of the most interesting characteristics. Birefringence in PCFs usually results from intentionally reducing the rotational symmetry of the fiber structure. The effect of the birefringence is more obvious if the index contrast between the core-cladding is high. Since great attention of air-silica PCFs, research on modal birefringence has thrived [12-14], partly due to the fact that structure symmetry can be easily destroyed in such high index-contrast PCFs.

As described in our previous work [15], the key point to induce high birefringence $B$ is to destroy the symmetry of the structure, and make the index difference between the two orthogonal polarization states. Usually, the anisotropy in PCFs can be introduced using elliptical air-holes [16-20] and with asymmetric core [12] or asymmetric distribution of air-holes in cladding [2]. In order to combine asymmetry in both the core and cladding, a PCF formed by a triangular lattice of elliptical air-holes with a double-defect as

\footnotetext{
* Corresponding author. Tel.: +886 3 3021878; fax: +88633411116

E-mail address: yfc01@cyu.edu.tw (Y.-F. Chau).
}

fiber core is reported to get $B \sim 10^{-2}$ in [12]. Based on the structure asymmetry, the PCF structure in [20] is proposed by employing elliptical air-holes in the fiber core (to induce high birefringence up to $10^{-2}$ order) but circular air-holes in the fiber cladding (to reduce the confinement loss). However, the shape of the elliptical air-holes needs a careful control in the fabrication process [21]. Ortigosa-Blanch et al. adopted asymmetric cladding design [2]; the PCF consists of triangular lattice air-holes with one adjacent central small air-hole missing (see Fig. 1c). Birefringence was introduced into the fiber only by a line defect of a small air-hole array in the PCF center.

The structures formed by the elliptical air-holes appear exhaustively complicated on the experience with fabrication of PCFs with varying hole sizes. Based on our previous works [15,19,20,22,23] and considered the drawback of the fabrication process (needs a careful control) of the elliptical air-hole PCF, in this paper, we report a new design of highly birefringent and low confinement loss PCF with complex unit cell cladding (see Fig. 1a and b) formed by the circular air-holes. Binary unit cell is the simplest way to form the complex cladding. The proposed structure has circular airholes of two different sizes to replace the singular size of air-holes in a PCF cladding, and is different from that proposed in conventional PCF designs $[2,3,24]$. Owing to the gaps between two different air-holes being larger than between of single holes, the corresponding fiber structure designed with our proposed complex air-holes in a PCF cladding is stronger than that with a singular size 

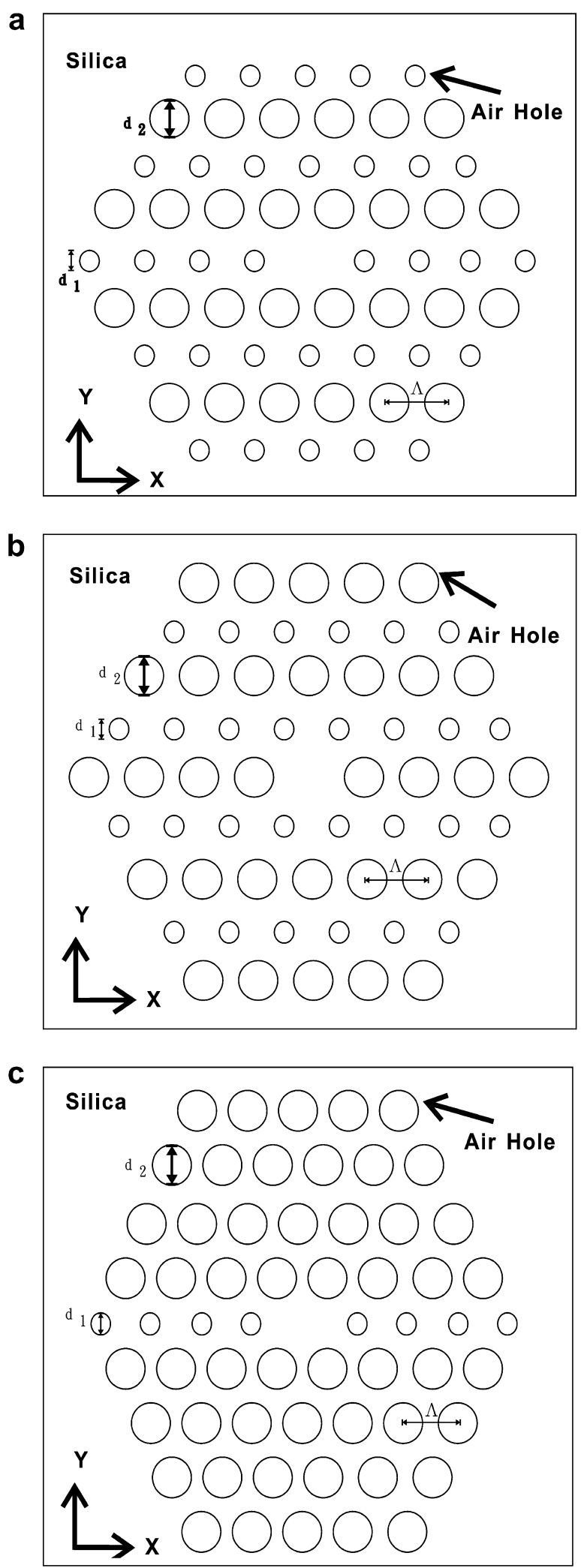

Fig. 1. Cross sections of birefringent PCFs. (a) Type 1 PCF, (b) Type 2 PCF, (c) previously proposed structure in Ref. [2].

of air-holes in conventional PCF designs under external forces. The birefringence of our structure is a result of the whole cladding asymmetry. The fiber core is a defect, which is formed by the omission of one circular air-hole in the PCF center, which the mode field is well confined in the core region; thus, it is possible to create a high birefrigence and low loss PCF. Together with the technological advancement in the fabrication of PCFs [25,26], it is possible to fabricate our suggested PCF, but the technology for forming the complex cladding (different sizes of small and large air-holes) may be some of the challenges expected during fabrication of the proposed fiber.

\section{Simulation method, models, results and discussions}

\subsection{Simulation method}

In this paper, we employ a full-vectorial finite-element method (FEM) $[27,28]$ with triangular elements for analyzing the birefringent PCFs. FEM simulations are performed by the commercial package Comsol Multiphysics 3.2b [29]. In order to evaluate confinement loss of the mode, an anisotropic perfectly matched layer [30] is employed as a boundary condition at computational domain edges.

\subsection{Structural parameters}

Two kinds of PCF cores (see Fig. 1a and b), which are formed by the omission of one air-hole of large size or small size in the center, are considered for comparison. For the convenience of statement, we name the corresponding fibers as Type 1 PCF (omitting small air-hole in the PCF center) and Type 2 PCF (omitting large air-hole in the PCF center). Our proposed PCF design is different from the previously proposed PCF designed in Ref [2] (see Fig. 1c). In the cross section of our designed structures, each lattice point forms the cladding, which consists of complex unit cell (two different sizes of circular air-holes). As shown in Fig. $1 a$ and $b, d_{1}$ and $d_{2}$ denote the diameters of the circular air-holes of small and large ones, respectively, and we define a parameter $\eta=d_{2} / d_{1}$. The fiber cladding consists of a triangular lattice of circular air-holes with a pitch (center to center distance between the different sizes of circular air-holes), $\Lambda=1.96 \mu \mathrm{m}$. The refractive index of the background material is set as $n=1.45$. The core is formed by the omission of one circular air-hole in the PCF center. For comparison with the previous structure shown in Fig. 1c, the effective air-hole area of types 1 and 2 is $A=\pi\left(d_{1}^{2}+d_{2}^{2}\right) / 8=\pi d_{3}^{2} / 4$, where $d_{3}$ is the diameter of large air-holes in the previous structure. In these cases, the air filling fractions in PCF claddings of Type 1, 2 and 3 PCF are almost the same.

\subsection{High-birefringence operation}

To understand the birefringent effect of the complex unit cell in the PCF cladding, we calculate the index of the fundamental spacefilling modes (FSMs) for the cladding photonic crystal using the conventional plane-wave expansion method. As expected, the FSMs in the cladding are split into approximately linear $y$ - and $x$ polarized states that are not degenerate. The calculated results are shown in Fig. 2. The index of the FSMs plays the role of an effective index for the cladding material, thus the complex unit cell cladding acts as a birefringent material. As seen from Fig. 2, the index of the $x$-polarized FSM is larger than that of the $y$-polarized one, and their difference reaches an order of $10^{-2}$ at lower normalized frequencies. Evidently, the high birefringence of whole fiber cladding will make Type 1 or Type 2 PCFs to be strongly birefringent. The birefringence of a PCF is determined by the difference between the effective indices of two orthogonal polarization modes

$B \equiv\left|n_{\mathrm{eff}}^{x}-n_{\mathrm{eff}}^{y}\right|$

where $n_{\text {eff }}^{x}$ and $n_{\text {eff }}^{y}$ are the refractive indices of the $x$ - and $y$-polarized fundamental modes of the PCF, respectively. 


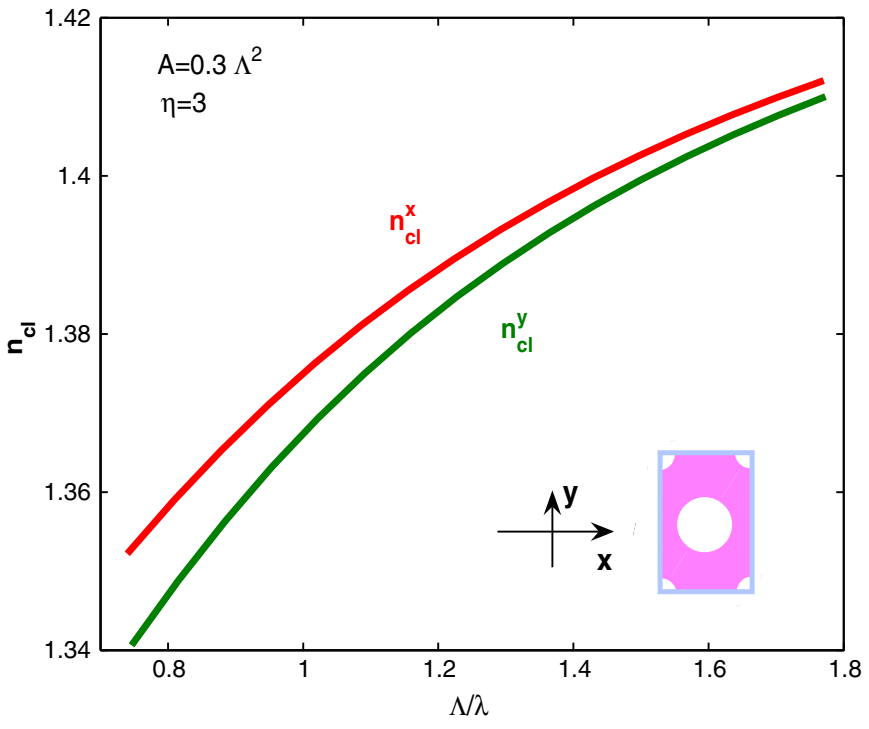

Fig. 2. The effective indices of the $x$ - and $y$-polarized FSM states as a function of normalized frequency $\Lambda / \lambda$. Inset shows the complex unit cell.

To illustrate the field profiles of our proposed PCFs, the fundamental modes of our designed Type 1 and Type 2 PCFs with effective air-hole area $A=0.3 \Lambda^{2}$ and $\eta=3$ at excitation wavelength $\lambda=1550 \mathrm{~nm}$, are shown in Fig. 3. It is clear from Fig. 3 that the modal field in Type 1 PCF is more strongly confined than the other one, due to that the core of Type 1 PCF is more effectively enclosed by the first ring of air-holes. The effective indices of the $y$ - and $x$ polarized modes for Type 1 PCF are $n_{\text {eff }}^{y}=1.402234$ and $n_{\text {eff }}^{x}=1.406442$, respectively, giving a birefringence $B \equiv$ $\left|n_{\text {eff }}^{x}-n_{\text {eff }}^{y}\right|=4.2 \times 10^{-3}$, which is much higher than that for the previous structure as shown in Ref. [2]. In addition, the birefringence obtained for Type 2 PCF (see Fig. 1b) is $B=1.2 \times 10^{-3}$, which is lower than those for the structures shown in Fig. 1a and $c$ and will be discussed later.

The effective index and birefringence of PCFs are relative to airhole sizes and operating wavelength (or normalized frequency $\Lambda$ / ג). Fig. 4 shows the birefringence for various PCF structures as a function of normalized frequency $\Lambda / \lambda$. It can be clearly seen that the birefringence is sensitive to the varying wavelength $\lambda$ and the air-hole sizes. As expected, the birefringent cladding can cause high birefringence in PCFs. In Fig. 4, we find that the birefringence is sensitive to the varying wavelength $\lambda$, and the birefringence in our proposed Type 1 PCF is higher than that in previous proposed structure when the value of $\Lambda / \lambda$ is larger than 0.9 . It can be clearly seen that the birefringence of our proposed Type $1 \mathrm{PCF}$ reaches its maximum value of $B=6 \times 10^{-3}$ at $\Lambda / \lambda=1.1$. The corresponding wavelength $\lambda=1.55 \mu \mathrm{m}$ occurs at the value of $\Lambda / \lambda=1.25$ with birefringence $B=4.2 \times 10^{-3}$ for our proposed Type $1 \mathrm{PCF}$, and the birefringence $B=1.61 \times 10^{-3}$ for previous proposed structure. It is evident from Fig. 4 that the field is confined in the point defect core at short wavelengths and field penetrates further into the asymmetry cladding region at long wavelengths. This phenomenon can also be explained by the following argument: The asymmetric cladding dominates at long wavelength, whereas the asymmetric core dominates at short wavelength. The reason why the birefringence of Type 2 PCF (see Fig. 1b) is smaller than the other two types (see Fig. 1a and c) is that the birefringent effects in the cladding and core are opposite. As can be seen from Fig. 2, the asymmetry cladding inclines the $x$-polarized mode of Type 2 PCF to have a larger index. On the other hand, the core of Type 2 PCF is ellipse-like with a major axis in the $y$-direction, which inclines the $y$-polarized mode to have a larger index.

As the cladding of the proposed PCFs is strongly asymmetry, it is necessary to analyze the stability of the birefringence of these PCFs. It should be noted that the claddings in our PCFs are strictly periodic, whereas the periodicity of the cladding in the previous PCF is broken by a line defect of small air-holes. We set the wavelength of $\lambda=1.55 \mu \mathrm{m}$, which corresponds to the operation wavelength in optical communication. In order to obtain a better visualization of the birefringence stability that occurs in our proposed fiber, the dependence of the modal birefringence on the number of the rings of air-holes surrounding the core is plotted in Fig. 5. It can be clearly seen in Fig. 5, the birefringence of our structure rapidly tends to be stable when the number of rings is larger than $N=4$. In contrast, the birefringence in the previous structure varies substantially even at $N=12$. Evidently, the stability of birefringence is closely related to the confinement of modal fields, and the modal field confinement is better in our structures, which will be demonstrated later in this paper.

Fig. 6 shows the simulated results of the birefringence as a function of effective air-hole area for three types of PCFs. The result shows that the birefringence increases with the effective air-hole

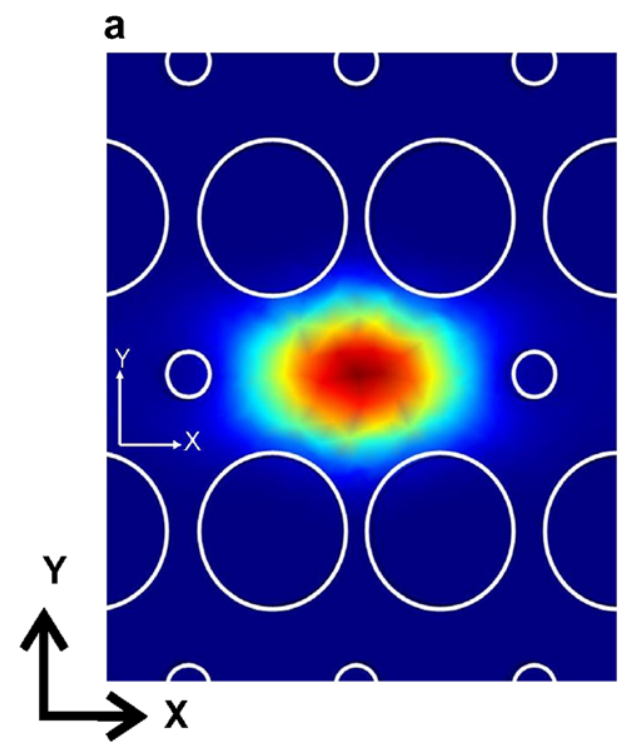

b

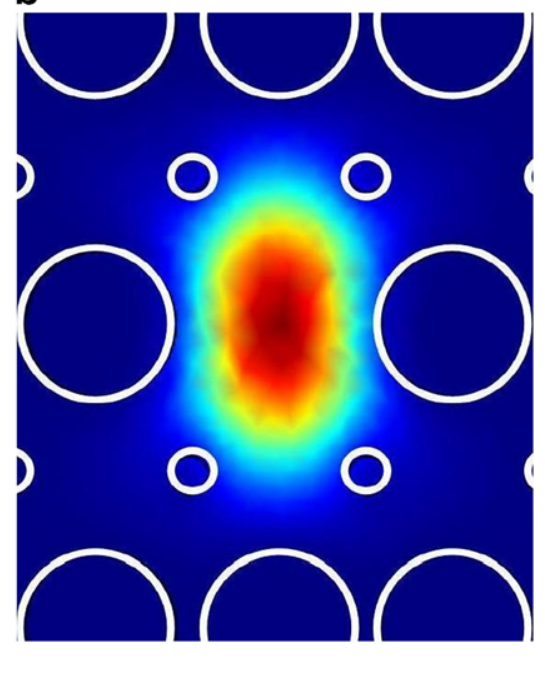

Fig. 3. Modal field pattern for two proposed PCFs with $\Lambda=1.96 \mu \mathrm{m}, A=0.3 \Lambda^{2}$ and $\eta=3$ at $\lambda=1550 \mathrm{~nm}$. (a) Type 1 PCF, (b) Type 2 PCF. 


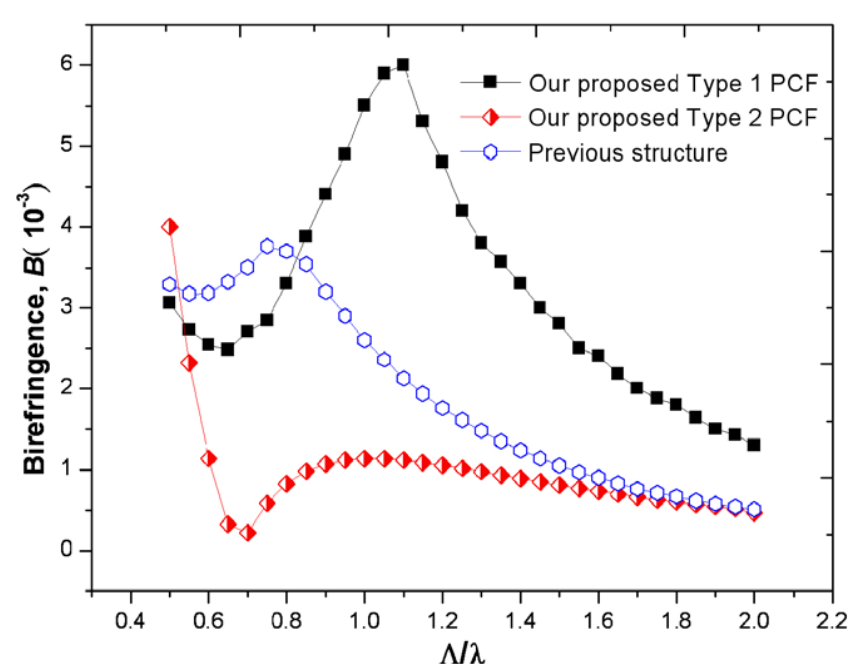

Fig. 4. Dependence of modal birefringence on normalized frequency for two proposed PCFs with $\eta=4$ and $A=0.3 \Lambda^{2}$. The corresponding results for the previous structure $(\eta=3)$ are included for comparison.

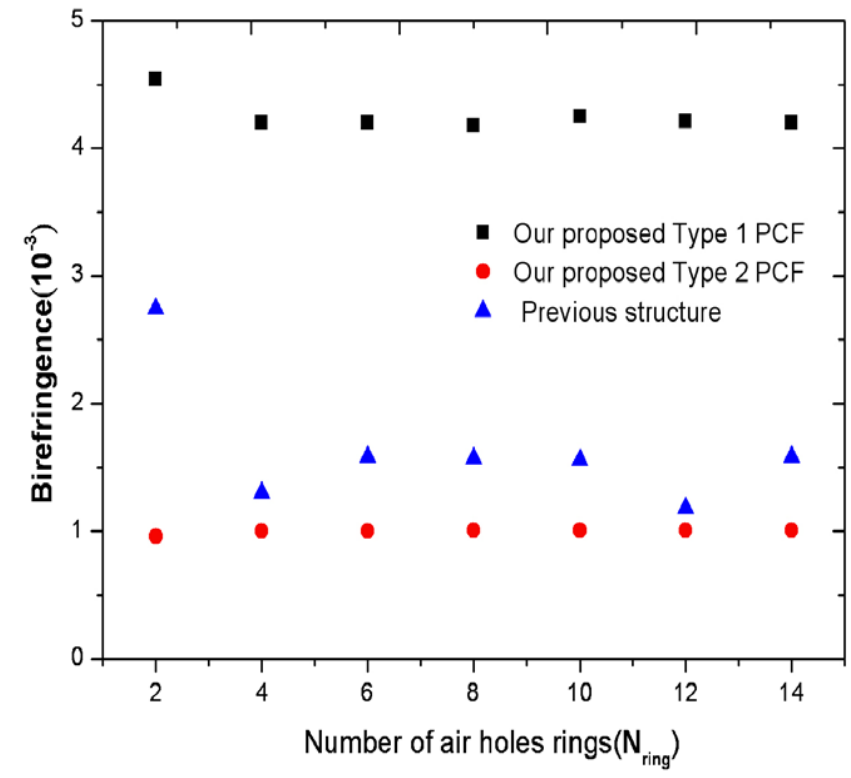

Fig. 5. Dependence of modal birefringence on the number of air hole rings for Type 1 , Type 2 , and previous PCFs at $\lambda=1550 \mathrm{~nm}$. The parameters are $\Lambda=1.96 \mu \mathrm{m}, \eta=4$ for Type 1 and 2 PCFs and $\eta=3$ for the previous structure.

area. With the increasing effective air-hole area, the asymmetry enhances in the cladding as well as in the core. As a consequence, the birefringence increases as the effective air-hole area increases. In contrast to the previous PCF shown in Fig. 1c, the birefringence of Type $1 \mathrm{PCF}$ is more sensitive to the variation of the effective airholes area. When the effective air-hole area $A / \Lambda^{2}$ is higher than 0.1 , the birefringence of our proposed Type 1 PCF is much higher than that for the previous structure. In Fig. 6, a birefringence of $B=8 \times 10^{-3}$ in our proposed Type 1 PCF is achieved for effective air-hole area $A / \Lambda^{2}=0.4$, indicating that the birefringence is nearly 3.6 times higher than that $\left(B=2.2 \times 10^{-3}\right)$ of previously proposed structure (see Fig. 1c). Our calculation indicates that the birefringence is higher than $6 \times 10^{-3}$ over a range of $\eta=2.6 \sim 6$ for our proposed Type 1 PCF with $A / \Lambda^{2}=0.4$.

Fig. 7 shows the birefringence of our proposed Type 1 PCF at various effective areas as a function of air-hole size ratio $\eta$. In this

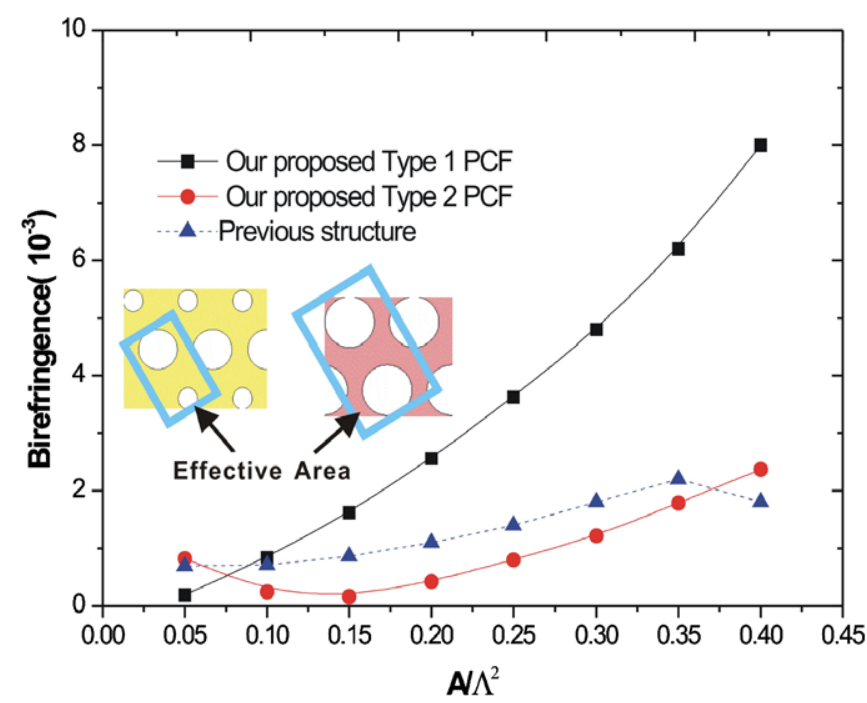

Fig. 6. Dependence of modal birefringence on effective air hole area, whereas $\Lambda=1.96 \mu \mathrm{m}, \eta=4$ for Type 1 and 2 PCF and $\eta=3$ for the previous structure. The operation wavelength is $\lambda=1550 \mathrm{~nm}$.

case, the confinement effect of the structure to the field becomes strong as the influence of the asymmetric cladding increases; thus, the birefringence increases. The birefringence behavior can be expected to be similar to that of radiation modes shown in Fig. 6. For each family of curves, the birefringence increases with the effective area is increased from $A / \Lambda^{2}=0.1$ to 0.42 , because the asymmetry of the structure increases. That is to say, the large effective area represents a strong perturbation to the field. Hence, the proper size of the air-holes can be chosen to achieve the highly Hi-Bi PCF. For our proposed Type 1 PCF with effective area $A / \Lambda^{2}$, a maximum value $B=8.7 \times 10^{-3}$ is reached at $\eta=4.5$, which is clearly larger than what is obtained from the corresponding designs reported in Refs. $[1,2]$.

The FEM is used to calculate the confinement loss of the fundamental modes in three types of PCFs, and results are plotted in Fig. 8. As an example of our interest, the parameters are $A / \Lambda^{2}=0.4$, $\eta=4.5$, and $\lambda=1550 \mathrm{~nm}$. As seen in Fig. 8, with the number of

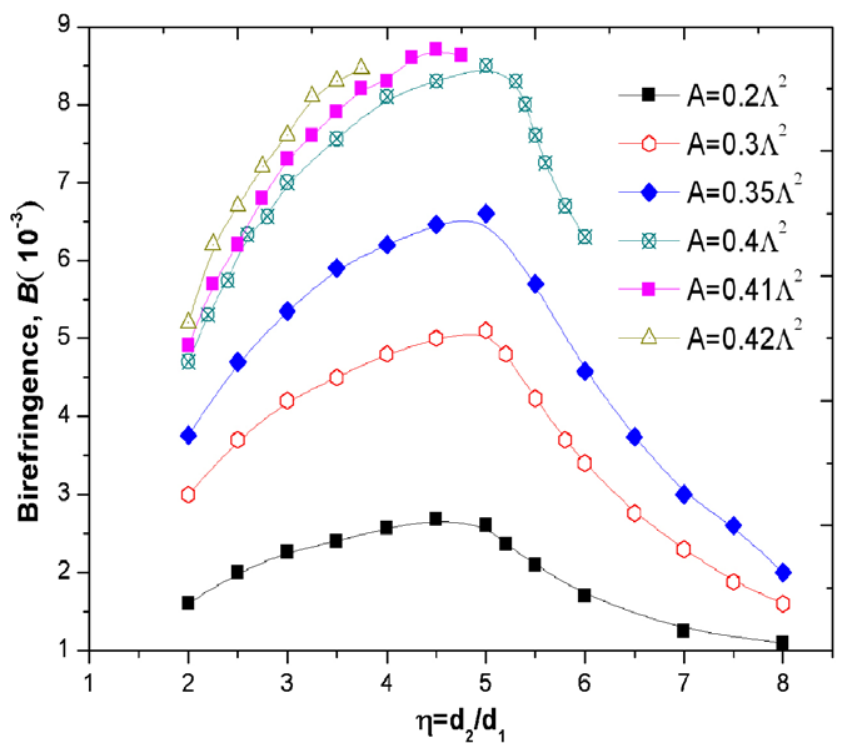

Fig. 7. Modal birefringence versus the parameter $\eta$ for the proposed Type 1 PCFs with various effective areas at $\lambda=1550 \mathrm{~nm}$. 


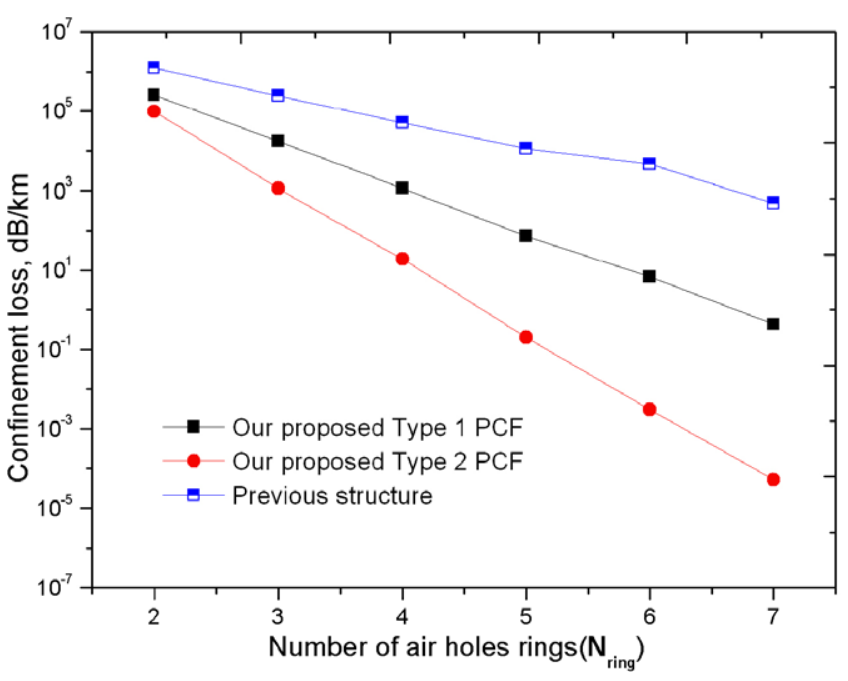

Fig. 8. Dependence of confinement loss on the number of air hole rings at $\lambda=1550 \mathrm{~nm}$ for Type 1 , Type 2 PCFs, and the previous PCF. The parameters are $\Lambda=1.96 \mu \mathrm{m}, A=0.4 \Lambda 2$, and $\eta=4.5$.

air-hole rings increasing, the confinement loss rapidly decreases, meaning that the confinement of the guided mode is significantly improved. The previous proposed PCF has the large confinement loss, whereas confinement loss for our Type 1 PCF is reduced at least one order, and further reduction can be achieved by Type 2 . The confinement losses of our proposed Type 1 and Type 2 PCF are 0.42 and $5.28 \times 10^{-5} \mathrm{~dB} / \mathrm{km}$, which is much lower than the value of $488.97 \mathrm{~dB} / \mathrm{km}$ for the previous structure, when the number of the air-hole rings is $N=7$. The reason why the confinement loss of our proposed PCF is much lower than the previously proposed PCF is that the core in our proposed PCF is better enclosed by the first ring of air-holes under the condition of identical effective air-hole area. So our proposed structure can achieve superior performance with high birefringence and low confinement loss under certain parameters.

\section{Conclusions}

We have successfully demonstrated high birefringence and low confinement loss PCF using point defect in core and complex unit cell in cladding. It has been shown that the cladding of our proposed PCFs is completely birefringent, though it is only formed by circular air-holes. The obtained results (birefringence $B=8.7 \times 10^{-3}$ ) show that the birefringence of our proposed Type $1 \mathrm{PCF}$ is much higher than the previous proposed structure studied in Refs. [1] (birefringence $B=2.35 \times 10^{-3}$ ) and [2] (birefringence $B=3.7 \times 10^{-3}$ ). Another merit of our proposed Type $1 \mathrm{PCF}$ is the low confinement loss, which is close to $5.28 \times 10^{-5} \mathrm{~dB} / \mathrm{km}$ for seven rings of air-holes in the PCF cladding. As a result, the design of forming the point defect by the omission of one air-hole in highly birefringent PCFs with whole asymmetry cladding is illustrated to reduce confinement loss and increase birefringence. As only circular air-holes are included in the structure, our proposed
PCF in this paper is easy to fabricate. For the future insight of examining the possibility of higher birefringence operation, we would like to study the impact of infiltrating the cladding holes with a common nematic liquid crystal material. Our simulation result is useful and provides a new concept for designing high birefringence and low loss PCF with good performance.

\section{Acknowledgment}

The authors are thankful for the financial support from the National Science Council, Taiwan, ROC, under Grant Number NSC-952119-M-009-029, NSC 96-2112-M-231-001-(1/3)-MY3 and NSC 96-2120-M-002-017. L.F. Shen also would like to thank the financial support by the Nature Science Foundation of China under contract Grant Number 60277018.

\section{References}

[1] Y. Jung, S.R. Han, Soan.Soan Kim, U.C. Paek, K. Oh, Opt. Lett. 31 (2006) 2681.

[2] A. Ortigosa-Blanch, J.C. Knight, W.J. Wadsworth, J. Arriaga, B.J. Mangan, T.A Birks, P.St.J. Russell, Opt. Lett. 25 (2000) 1325.

[3] T.A. Birks, J.C. Knight, P.St.J. Russell, Opt. Lett. 22 (1997) 961.

[4] J.K. Ranka, R.S. Windeler, A.J. Stentz, Opt. Lett. 25 (2000) 796.

[5] Tuomo.Tuomo Ritari, H. Ludvigsen, M. Wegmuller, M. Legré, N. Gisin, J. Folkenberg, M. Nielsen, Opt. Express 12 (2004) 5931.

[6] M. Nielsen, N. Mortensen, M. Albertsen, J. Folkenberg, A. Bjarklev, D. Bonacinni, Opt. Express 12 (2004) 1775.

[7] M. Nielsen, C. Jacobsen, N. Mortensen, J. Folkenberg, H. Simonsen, Opt. Express 12 (2004) 1372.

[8] Jacob.Jacob Folkenberg, M. Nielsen, N. Mortensen, C. Jakobsen, H. Simonsen, Opt. Express 12 (2004) 956.

[9] N.A. Mortensen, J.R. Folkenberg, M.D. Nielsen, K.P. Hansen, Opt. Lett. 28 (2003) 1879.

[10] M.D. Nielsen, N.A. Mortensen, J.R. Folkenberg, A. Bjarklev, Opt. Lett. 28 (2003) 2309.

[11] K. Saitoh, M. Koshiba, IEEE Photon. Technol. Lett. 15 (2003) 1384.

[12] T.P. Hansen, J. Broeng, S.E.B. Libori, E. Knudsen, A. Bjarklev, J.R. Jensen, H. Simonsen, IEEE Photon. Technol. Lett., 13 (2001) 588.

[13] K. Suzuki, H. Kubota, S. Kawanishi, M. Tanaka, M. Fujita, Opt. Express, 9 (2001) 676.

[14] H. Kubota, S. Kawanishi, S. Koyanagi, M. Tanaka, S. Yamaguchi, Lett. 16 (2004) 182.

[15] Y.-F. Chau, H.-H. Yeh, D.P. Tsai, Jpn. J. Appl. Phys. 46 (2007) L1048.

[16] M.J. Steel, R.M. Osgood Jr., J. Lightwave Technol. 19 (2001) 495.

[17] J. Broeng, D. Mogilevtsev, S.E.B. Libori, A. Bjarklev, in: Optical Fiber Communication Conference, 54 of OSA Trends in Optics and Photonics, Optical Society of America, Washington, DC, 2001, paper MA1.3.

[18] M.J. Steel, R.M. Osgood Jr., Opt. Lett. 26 (2001) 229.

[19] Daru.Daru Chen, Linfang.Linfang Shen, J. Lightwave Technol. 25 (2007) 2700.

[20] Daru.Daru Chen, Linfang.Linfang Shen, IEEE Photon. Technol. Lett., 19 (2007) 185.

[21] Partha Roy.Roy Chaudhuri, Varghese.Varghese Paulose, Chunliu.Chunliu Zhao, Chao.Chao Lu, IEEE Photon. Technol. Lett., 16 (2004) 1301.

[22] Y.-S. Sun, Y.-F. Chau, H.-H. Yeh, T.-J. Yang, D.P. Tsai, Appl. Opt. 46 (2007) 5276

[23] Yuh-Sien Sun, Yuan-Fong Chau, Han-Hsuan Yeh, Din Ping, Jpn. J. Appl. Phys. 47(5), inpress.

[24] J.C. Knight, T.A. Birks, P.St.J. Russell, D.M. Atkin, Opt. Lett. 21 (1996) 1547.

[25] Vito.Vito Mocella, Opt. Express 13 (2005) 1361.

[26] A.N. Grigorenko, A.K. Geim, H.F. Gleeson, Y. Zhang, A.A. Firsov, I.Y. Khrushchev, J. Pertrovic, Nature 438 (2005) 35.

[27] A. Proulx, J.-M. Ménard, N. Hô, J.M. Laniel, R. Vallée, C. Paré, Opt. Express, 11 (2003) 3338.

[28] W.V. Sorin, B.Y. Kim, H.J. Shaw, Opt. Lett. 11 (1986) 581.

[29] http://www.comsol.com/.

[30] Kunimasa.Kunimasa Saitoh, Masanori.Masanori Koshiba, IEEE J. Quantum Elect. 38 (2002) 927. 